\title{
The coordinate-tracking detector based on the drift chambers
}

\author{
A.A. Petrukhin ${ }^{1, a}$, N.V. Ampilogov ${ }^{a}$, N.S. Barbashina ${ }^{a}$, A.G. Bogdanov ${ }^{a}$, \\ A.A. Borisov ${ }^{a, b}$, D.V. Chernov ${ }^{a}$, L.I. Dushkin ${ }^{a}$, R.M. Fakhrutdinove, ${ }^{a}$, \\ R.P. Kokoulin ${ }^{a}$, K.G. Kompaniets ${ }^{a}$, A.S. Kozhin ${ }^{a, b}$, V.V. Ovchinnikova, \\ A.S. Ovechkin ${ }^{a}$, V.A. Selyakov ${ }^{a}$, V.V. Shutenko ${ }^{a}$, N.S. Volkov ${ }^{a}$, I.I. Yashin ${ }^{a}$, \\ E.A. Zadeba ${ }^{a}$ \\ a National Research Nuclear University MEPhI (Moscow Engineering Physics Institute), 115409 \\ Moscow, Russian Federation \\ ${ }^{b}$ RF SSC Institute of High Energy Physics, 142281, Protvino, Russian Federation \\ E-mail: AAPetrukhin@mephi.ru
}

The large-scale coordinate-tracking detector for registration of near-horizontal muon flux generated by ultra-high energy cosmic rays is being developed in MEPhI. Detector is based on the multiwire drift chambers from the neutrino experiment at the IHEP U-70 accelerator. Their key advantages are a large effective area $\left(1.85 \mathrm{~m}^{2}\right)$, good coordinate and angular resolution with a small number of measuring channels. Detector will be operated as a part of the experimental complex NEVOD, in particular, jointly with Cherenkov water detector (CWD) with volume of 2000 cubic meters and coordinate detector DECOR. The prototype of this detector (CTUDC) representing two coordinate planes of 8 drift chambers in each has been developed and has been mounted on the opposite sides of CWD. The prototype has the same principle of joint operation with NEVOD-DECOR triggering system and the same drift chambers arrangement, so main features of the full detector can examined. Results of a cross-calibration of the CTUDC and coordinate-tracking detector DECOR and a joint operation with the NEVOD-DECOR complex are presented.

The 34th International Cosmic Ray Conference

30 July - 6 August, 2015

The Hague, The Netherlands

${ }^{1}$ Speaker 


\section{Introduction}

The project aim is solving the problem of the excess of muon bundles that increases with the energy of the primary cosmic rays $[1,2]$, what can be caused by both astro- or nuclearphysical reasons [3]. The only characteristics that responds differently to changes in the composition of cosmic rays and the inclusion of new physical processes, but has not been investigated to the present time, is the energy of the muon component of extensive air showers [3]. Such studies are performed at the experimental complex NEVOD-DECOR [4]; however, the coordinate detector DECOR does not cover the entire aperture of the water Cherenkov detector and does not exclude the possibility of passing of part of muons between the individual supermodules of the detector. Besides, the size of its cells limits the possibility of separating two or more particles at small distances (less than $3 \mathrm{~cm}$ ). The new coordinate-tracking detector [5] based on drift chambers will increase the coverage of the side aperture of the Cherenkov water detector (CWD) NEVOD and significantly improve the resolution of close tracks.

The detector is based on the multiwire drift chambers from the neutrino experiment at the IHEP U-70 accelerator [6]. The specific features of such experiment (low event rate, moderate requirements for spatial resolution) allow the use of drift chambers with a large drift gap. The chamber characteristics (large effective area and good resolution with just 4 measuring channels) perfectly match the goals of the project of the new coordinate-tracking detector.

Overall sizes of the chamber are $4000 \times 508 \times 112 \mathrm{~mm}^{3}$. Sensitive area is $1.85 \mathrm{~m}^{2}$ that is $91 \%$ of the chamber area. The chamber represents an aluminum box with ends limited by plexiglas plugs, to which wires, gas inlets and high voltage connectors are mounted. A uniform electric field is formed by field-forming wires with a pitch of $5 \mathrm{~mm}$, the potential on them varies linearly from 0 to $12 \mathrm{kV}$. The drift chamber is filled with a gas mixture of $94 \% \mathrm{Ar}$ and $6 \% \mathrm{CO}_{2}$; at field strength of $480 \mathrm{~V} / \mathrm{cm}$ electron drift velocity is constant $(\sim 41 \mathrm{~mm} / \mu$ s for the gas mixture) and allows to use linear time/coordinate relation. The drift chamber has four signal wires alternately shifted by $\pm 0.75 \mathrm{~mm}$ in the drift direction relative to the center of the chamber. Gas amplification is generated by potential difference of $2.2 \mathrm{kV}$ between the signal and ten cathode wires arranged symmetrically at a distance of $3 \mathrm{~mm}$ from the center of the chamber. Two guard wires eliminate the edge effects.

With the passage of a charged particle through the working volume of the chamber, drift times of electrons to the signal wires are registered; taking into account the drift velocity of electrons, these times allow to reconstruct the projection of the track on the plane perpendicular to the wires. Thus, for the reconstruction of tracks in space at least two non-parallel drift chambers are required. The signals from the wires are processed by an on-board amplifiershaper that forms 50 to $100 \mathrm{~ns}$ pulses (depending on the signal length) in the LVDS levels. Spatial accuracy of the chamber is $1 \mathrm{~mm}$, the angular resolution is about $0.03 \mathrm{rad}$, two close tracks can be separated at a distance of $3 \mathrm{~mm}$. 


\section{CTUDC}

Coordinate-tracking unit based on drift chambers (CTUDC) consists of two vertical coordinate planes installed at the different sides of the CWD in the short galleries of the third floor of the NEVOD building, one floor above DECOR supermodules (Figure 1). Such location allows the registration of near horizontal tracks as by CTUDC (triggered by CWD) so by joint operation with DECOR, that will significantly increase the range of muon track zenith angles from $85-95^{\circ}$ to $80-100^{\circ}$.

Each plane consists of 8 drift chambers installed in two rows, overlapped by $30 \mathrm{~cm}$ to exclude dead zone in the chamber ends; it causes a $4^{\circ}$ angle between planes and CWD wall. The effective area of the plane is $14.8 \mathrm{~m}^{2}$, area of two DECOR supermodules located a floor below is $17.5 \mathrm{~m}^{2}$, so the total area of coordinate detectors for registration of near-horizontal particles coming along the CWD almost doubles. One of the main goals of this setup is to examine conditions of the joint operation with CWD and DECOR.

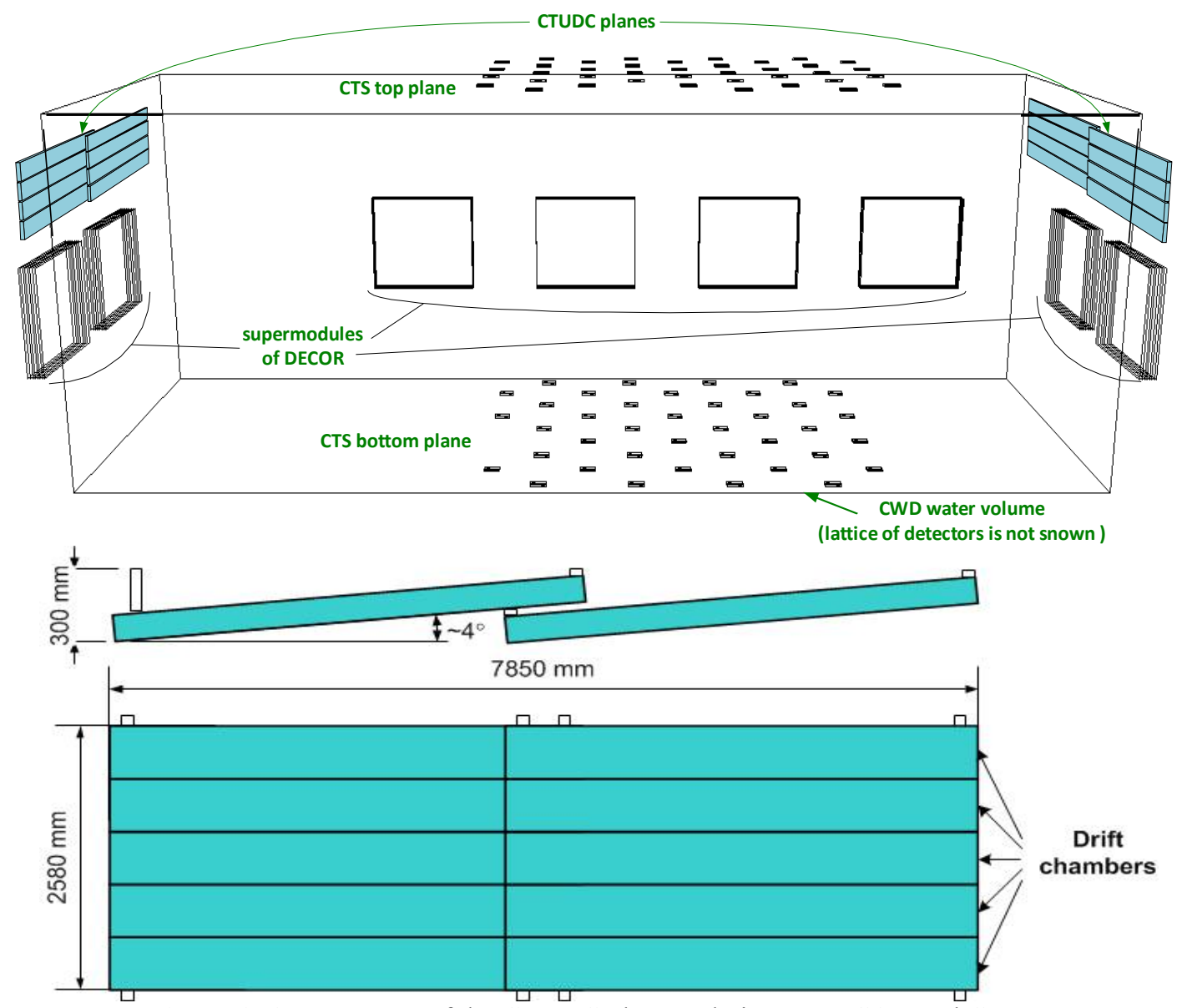

Figure 1: Arrangement of the CTUDC planes relative to DECOR and CWD.

\section{Registration system}

CTUDC is designed for joint operation with the triggering system of the experimental complex NEVOD (NEVOD TS), DAQ and primary data processing of CWD and DECOR. 
Triggering system has a rather fast data handling: the period between the passage of a particle through the working volume of CWD and the trigger formation is about $500 \mathrm{~ns}$. On the other hand, the maximum drift time of the electrons in the drift chamber is $6 \mu \mathrm{s}$, so registration system and DAQ of CTUDC cannot be directly integrated into the NEVOD TS and should be implemented separately. The Figure 2 shows the workflow of the CTUDC registration system.

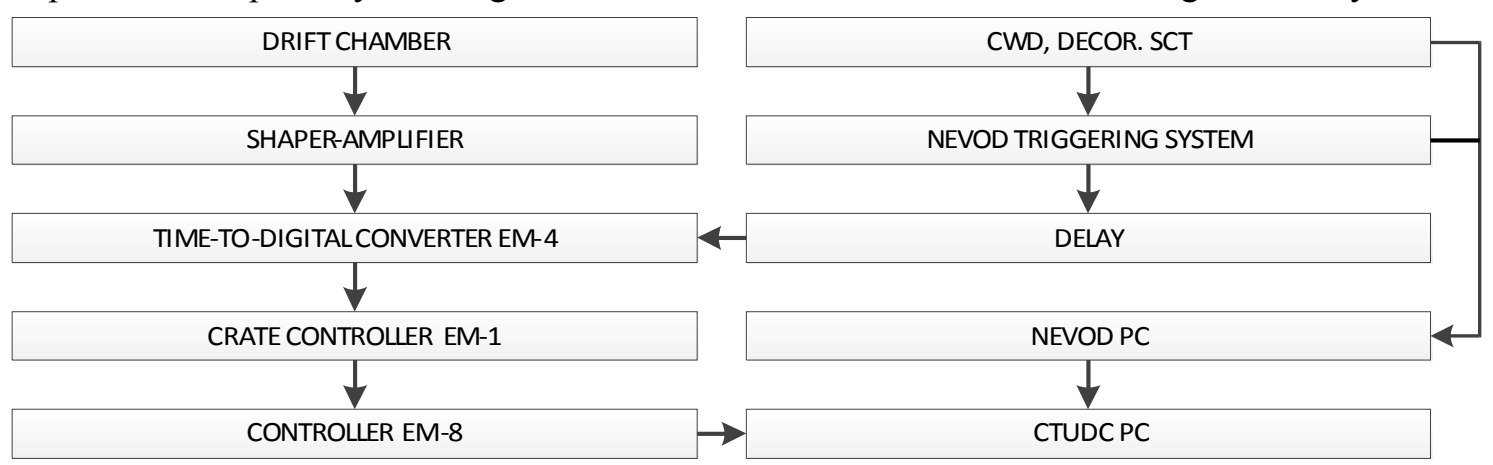

Figure 2: Workflow of the CTUDC registration system.

Primary processing of signals from sense wires of drift chambers is carried out by the 4-channel shaper-amplifier AMR-4 (mounted on the end face of the DC) with a single adjustable threshold for all channels. It forms $75 \mathrm{~ns}$ LVDS pulses that pass through several commutation blocks to a 128-channel time-to-digital converter EM-4. There are 64 channels in 16 chambers, and a single TDC can handle all of them, so after the expansion of the drift chamber setup to the TREK [6], CTUDC channels can be included into its registration system.

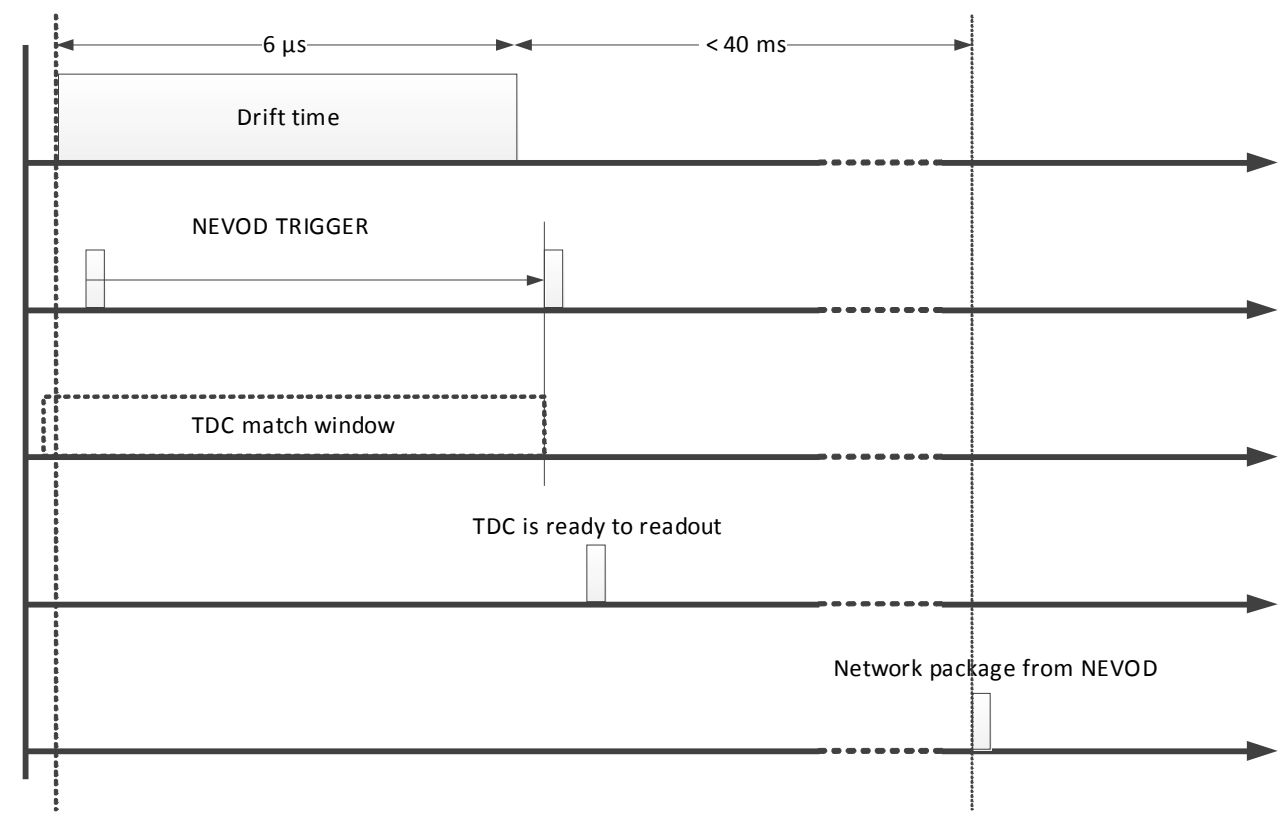

Figure 3: Timing diagram of the CTUDC registration system.

Trigger signal of the NEVOD TS acts as the time stamp for TDC, it is produced with implementation of any trigger condition of CWD and DECOR (configured separately for each detector). All delays in the triggering system are finely tuned, so a time between the passage of the first particle in the event and the all-system trigger does not depend on the type of completed trigger condition and is approximately $225 \mathrm{~ns}$, it takes $100 \mathrm{~ns}$ for cable delays and hardware jitters and $125 \mathrm{~ns}$ for the triggering system. All signals from the drift chambers should be 
received by the TDC before the arrival of the NEVOD trigger, so the delay of the trigger is necessary. For this purpose, a special block for VME crate was developed, it combines galvanic isolation by rapid optocoupler, TTL to NIM converter (MC10124P) and $8 \mu$ s delay (SN74123N).

After the generation of the trigger signal in the triggering system, the system transmits all its data to the NEVOD central computer that starts (via Ethernet) to gather all amplitude information from CWD and configuration of triggered DECOR streamer tubes. Simultaneously the NEVOD central computer sends a network packet to CTUDC that contains main information about the event: the number and time of the event, types of CWD and DECOR inner trigger signals. The network packet is started with a 4-byte sequence at which the CTUDC central computer determines the type of the received packet, decodes the data and starts to collect information from EM-4 TDC. Figure 3 shows timing diagram of the CTUDC registration system. After the primary data processing, the software of CTUDC central computer forms the event; later these events will be off-line joined with the events saved at the NEVOD central computer by means of time stamps and event numbers.

\section{First results of joint operation of CTUDC, CWD and DECOR}

The selection of events according to the type of the trigger in the NEVOD TS allows to investigate the temporal characteristics for each type of CWD and DECOR events. Figure 4 shows (on the left) the distribution of drift times of electrons in one of the drift chambers for events triggered by CWD. The width of the distribution is about $6000 \mathrm{~ns}$ that corresponds to the maximum drift time of electrons. This indicate a good stability of generation of the trigger signal from CWD. Figure 4 (on the right) shows the distribution of the number of muons registered by CTUDC plane in these events.
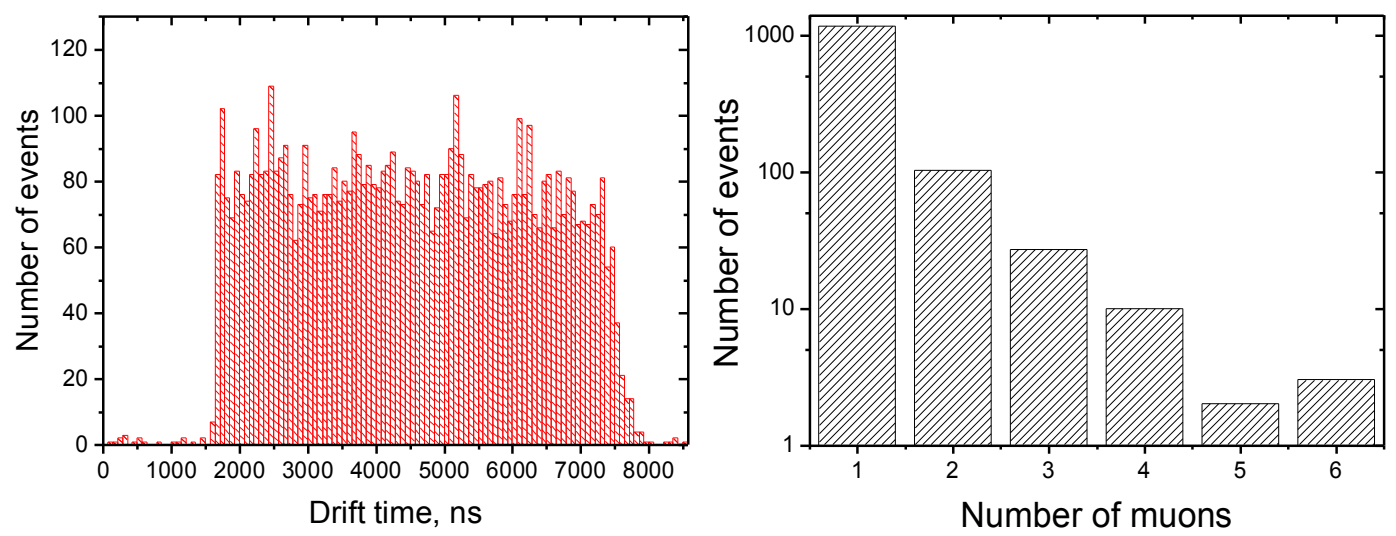

Figure 4: Distribution of events according to the drift time (left) and the multiplicity of muons (right) with the NEVOD trigger.

A new trigger condition was used for cross-calibration of the CTUDC drift chambers and DECOR supermodules: it chooses events, which have triggered the CWD and at least one supermodule. The muons in these events are moving at zenith angles ranging from $78^{\circ}$ to $88^{\circ}$ producing Cherenkov light to the most part of the CWD lattice. Rate of such events was about $0.2 \mathrm{~s}^{-1}$. 


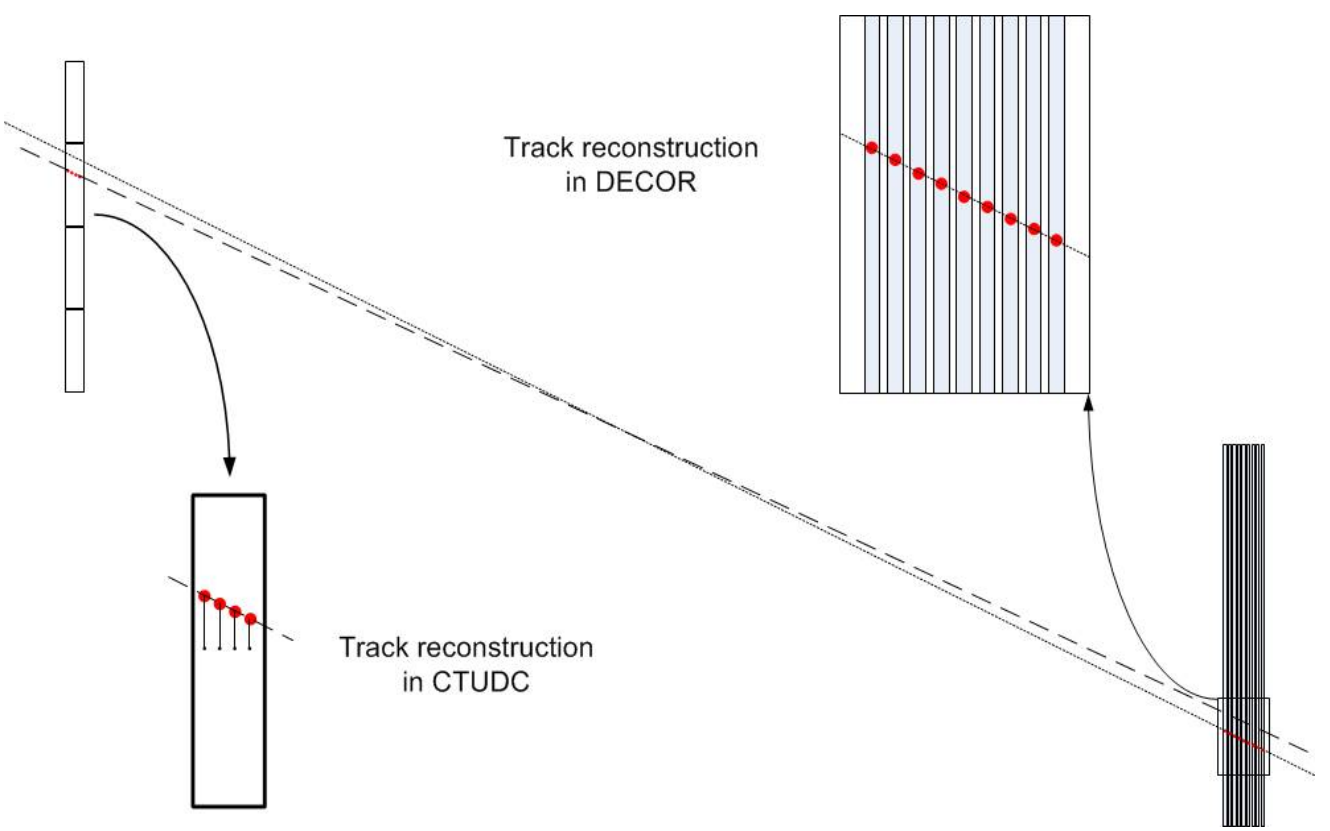

Figure 5: The principle of registration and track reconstruction for a joint operation of CTUDC and DECOR.

Figure 5 shows the principle of registration and track reconstruction by the joint operation of CTUDC and DECOR. Figure 6 shows the distribution in the difference in the muon track zenith angle estimates according to CTUDC and DECOR reconstruction. Taking into account the scattering of the muon in $26 \mathrm{~m}$ of water and $1.2 \mathrm{~m}$ concrete, as well as errors in the reconstruction of the angle in DECOR supermodules and drift chambers, the $5^{\circ} \mathrm{FWHM}$ can be considered as a successful proof of the efficiency of the coordinate-tracking detector CTUDC.

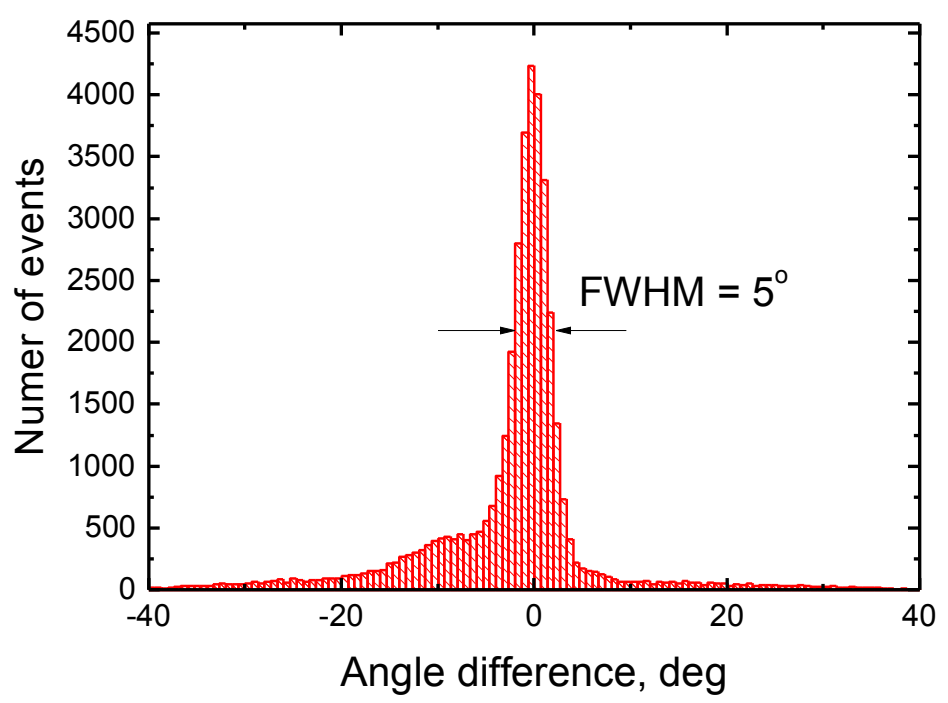

Figure 6: Difference in zenith angles of tracks reconstructed in DECOR and CTUDC. 


\section{Conclusion}

The unique coordinate-tracking detector CTUDC based on drift chambers from IHEP neutrino experiment is developed in MEPhI. Detector is operated jointly with other systems of the experimental complex NEVOD and is aimed at registration of near-horizontal flux of muons generated by ultra-high energy primary cosmic rays.

Currently the detector is on the calibration stage. It has been already cross-calibrated with the coordinate detector DECOR and works jointly with the Cherenkov water detector. First results of these tests showed a good performance of the detector.

This work is supported by the grant of the Russian Foundation for Basic Research (project 13-02-12207-ofi-m-2013).

\section{References}

[1] N.S. Barbashina et al., Ultra-high energy cosmic ray investigations by means of EAS muon density measurements. Nucl. Phys. B (Proc. Suppl.) 165 (2007) 317.

[2] A.G. Bogdanov et al., Investigation of the properties of the flux and interaction of ultrahigh-energy cosmic rays by the method of local-muon-density spectra. Physics of Atomic Nuclei 73 (2010) 852.

[3] A.A. Petrukhin, NEVOD-DECOR experiment and evidences for quark-gluon plasma in cosmic rays. Nucl. Phys. B (Proc. Suppl.) 212-213 (2011) 235.

[4] E.A. Kovylyaeva et al., Energy release of inclined muon groups in the nevod water Cherenkov detector Bull. Lebedev Phys. Inst. 41 (2014) 218.

[5] E.A. Zadeba et al., The coordinate-tracking detector based on the drift chambers for ultrahigh-energy cosmic ray investigations. Journal of Instrumentation 9 (2014) C08018.

[6] N.I. Bozhko et al., Drift chamber for the Serpukhov neutrino detector. Nucl. Instr. Meth. Phys. Res. A 243 (1986) 388. 